\title{
Analysis of STT-RAM Cell Design with Multiple MTJs Per Access
}

\author{
Henry Park, Richard Dorrance, Amr Amin, Fengbo Ren, Dejan Marković, and C.K. Ken Yang \\ Department of Electrical Engineering \\ University of California, Los Angeles \\ Los Angeles, CA 90095 \\ henrypark@ucla.edu
}

\begin{abstract}
Density of STT-RAMs is limited by the area cost and width of the access device in a cell since it needs to support the programming currents. This paper explores a cell structure that shares each cell's access transistor with multiple MTJ memory elements. Feasibility and limitations of such a cell structure is explored for both reading and writing of the memory. The analytical and simulation results indicate that only small amount of sharing is possible and having MTJs that can handle a high read current without disturbing the cell is needed.
\end{abstract}

Keywords-component; STT-RAM, Magnetic Tunnel Junction, $1 T-3 M T J$

\section{INTRODUCTION}

Aggressive integration of on-chip memory arrays raises the need for high-density memories. Advances in spin-torque transfer RAM (STT-RAM) enable storing data in a magnetic tunneling junction (MTJ) comprising a thin insulator and ferromagnetic sheets. The technology has shown promise as dense arrays of MTJs have been fabricated with very small cell sizes $[1,2]$. However, as shown in Fig. 1, due to the required junction current density for switching, the access transistor must be sized to support such currents. For technology nodes down to $32 \mathrm{~nm}$, the actual cell size is determined by the access transistor. This paper analyzes the effectiveness and challenges in building an STT-RAM memory cell that shares multiple MTJs in a cell with a single access transistor.

\section{BACKGROUND}

Similar to most memories, an STT-RAM typically employs a structure with one MTJ switched/accessed by a single access transistor (1T-1MTJ). The organization of a conventional STTRAM is shown in Fig. 2(a). The access transistor is sized [3] to meet the minimum write current requirement for the cell to switch either in the parallel to anti-parallel, $\min \left(\mathrm{I}_{\mathrm{W}}(\mathrm{AP} \rightarrow \mathrm{P})\right)$, or vice versa, $\min \left(\mathrm{I}_{\mathrm{W}}(\mathrm{P} \rightarrow \mathrm{AP})\right)$. Directions of each switching current are depicted in Figure 3. Switching from AP to $P$ is equivalently moving operating point from A to B in Fig. 3(a) while switching in reverse direction (P to AP) is from $\mathrm{C}$ to $\mathrm{D}$ in Fig. 3(b). In both cases current density can be improved by boosting voltages above the nominal supply level [4]. Since each cell has a dedicated access device, a cell's value is not perturbed by the writing of other cells. As long as the maximum read current, $\max \left(\mathrm{I}_{R}(\mathrm{AP})\right)$ or $\max \left(\mathrm{I}_{\mathrm{R}}(\mathrm{P})\right)$, provides sufficient margin from the minimum write currents, the cell

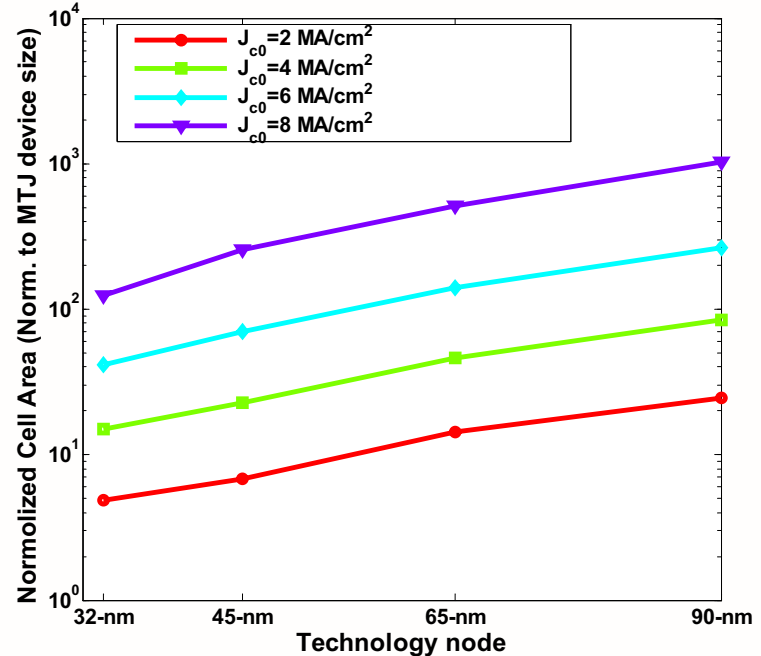

Fig. 1. Required normalized NFET cell area at different technology nodes $\left(\mathrm{RA}=4.9 \Omega \cdot \mu \mathrm{m}^{2}, \mathrm{TMR}=120 \%\right.$, MTJ size $=70 \times 120 \mathrm{~nm}^{2}$

remains stable during a read operation (we assume minimum write current at $99.99 \%$ switching probability while maximum read current is at $0.01 \%$ ). Such reliable read operation is traded with scalability of the memory cell due to minimum NFET size (Fig. 1). This paper explores possibility of sharing multiple MTJs with a single access device to reduce its impact on the cell size.

\section{DESIGN CONSIDERATIONS}

Structure of multiple MTJs per cell is shown in Fig. 2(b). The figure shows a simple case of two MTJs per single access NFET, but theoretically more MTJ devices could share a single NFET. While improving array density, this structure introduces additional complexity and limitations in both reading and writing. In general, when one cell is accessed, current can flow between the bit-lines through resistive paths formed by nonaccessed cells. Fig. 4 shows such an example. This parasitic current results in both degraded read and unstable non-accessed cells during writes. It may also limit the number of cells in a column hence leading to a finer sub-array partitioning of the memory. Similar analysis can be found from papers of crosspoint (CP) cell MRAM (Magnetic Random Access Memory), where the authors removed the access device to minimize the

Sponsored by the DARPA/MTO STT-RAM program 


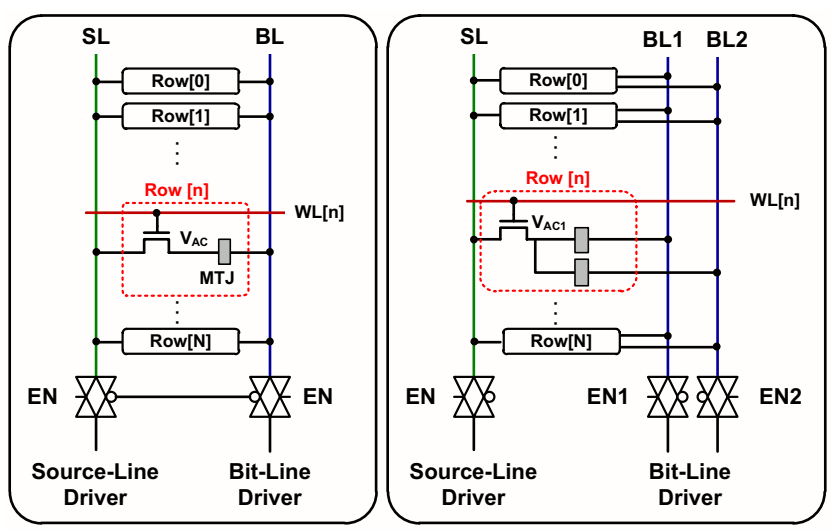

Fig. 2. (a) Standard 1T-1MTJ Structure (left). (b) Suggested 1T-2MTJ Structure with separated bit lines (right).

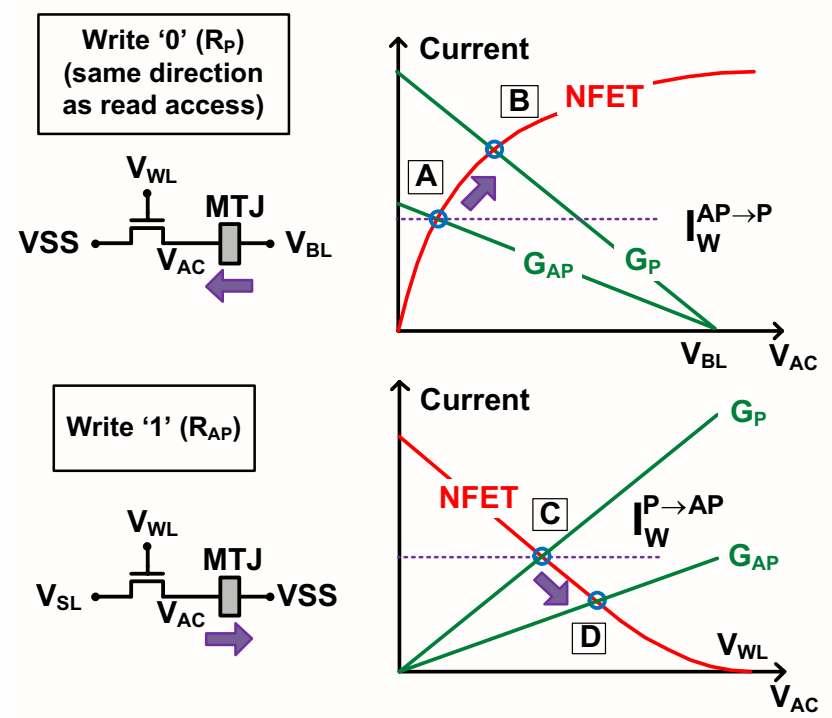

Fig. 3. Load line of NFET and MTJ device ( $G$ denotes conductance) in case of (a) reading or writing $\mathrm{R}_{\mathrm{P}}$ (above) and (b) writing $\mathrm{R}_{\mathrm{AP}}$ (below).

cell area smaller than $6 \mathrm{~F}^{2}$ but they also revealed limitations on the array size and MTJ characteristics [5 7].

\section{A. Impact of MTJ Sharing on Reads}

Fig. 4 can be used to illustrate when a single MTJ in a cell is being read. The total amount of read current is the sum of the main MTJ current ( $\mathrm{I}_{\mathrm{MTJ}}$ through MTJ1 in the Fig. 4) and the parasitic current through all other MTJs attached to its bit-line (BL1). If the number of MTJ devices per cell is $\mathrm{N}_{\mathrm{MTJ}}$ and the number of cells per column is $\mathrm{N}_{C}$, then the equivalent parasitic resistance between $\mathrm{BL} 1$ and $\mathrm{V}_{\mathrm{ACl}}$ are expressed as [7]

$$
\boldsymbol{R}_{P A R}=\frac{\left(N_{M T J}+N_{C}-1\right)}{\left(N_{M T J}-1\right) \cdot\left(N_{C}-1\right)} R_{M T J}=A_{P A R} \cdot \boldsymbol{R}_{M T J}
$$

$R_{M T J}$ is a discrete random variable bounded by $R_{P}$ and $R_{A P}$. In comparison to a $1 \mathrm{~T}-1 \mathrm{MTJ}$ cell, the parasitic resistive paths reduce the resistance difference when reading either $\mathrm{R}_{\mathrm{P}}$ or $\mathrm{R}_{\mathrm{AP}}$ of MTJ1. The parasitic paths effectively reduce the TMR seen

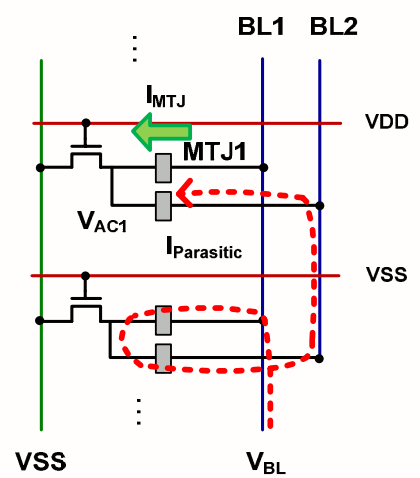

Fig. 4. Illustration of parasitic current disturbance. $\mathrm{I}_{\mathrm{MTJ}}$ is the wanted current through the selected device.
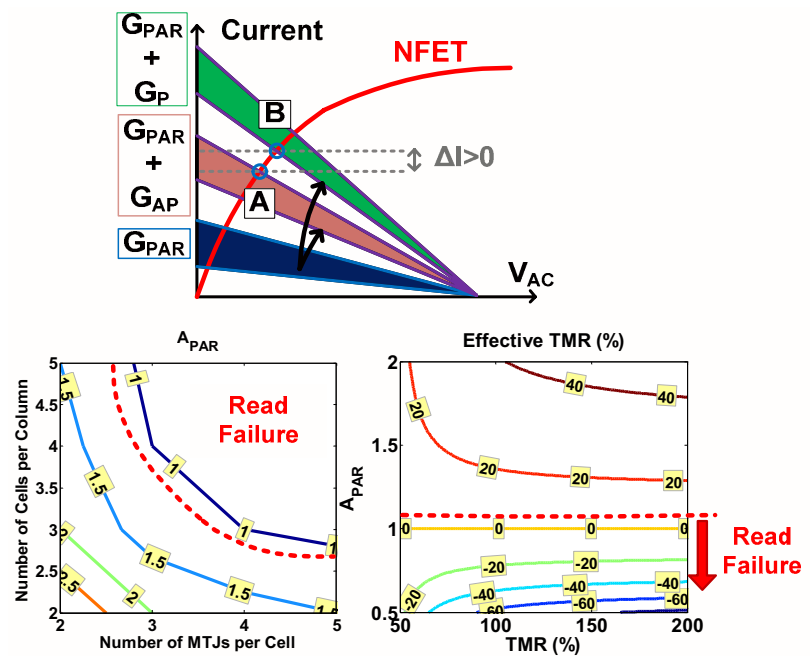

Fig. 5. (a) Load line of NFET and MTJs during read access (upper). (b) $A_{P A R}$ as function of the number of MTJs per cell and the number of cells per column (lower left). (c) Effective $T M R$ as a function of $A_{P A R}$ and TMR (lower right).

by the read sense amplifier and an effective TMR can be expressed as

$$
\begin{aligned}
\boldsymbol{T M R _ { e f f } =} & \frac{\boldsymbol{R}_{A P}\left\|A_{P A R} \cdot \boldsymbol{R}_{P}-\boldsymbol{R}_{P}\right\| A_{P A R} \cdot \boldsymbol{R}_{A P}}{\boldsymbol{R}_{P} \| \boldsymbol{A}_{P A R} \cdot \boldsymbol{R}_{A P}} \\
& =\frac{\left(A_{P A R}-1\right) \cdot \boldsymbol{T M R}}{\left(A_{P A R} \cdot(1+\boldsymbol{T M R})+1\right)}
\end{aligned}
$$

Fig. 5(a) illustrates the impact of the parasitic resistance on current load lines from an MTJ resistance loading the nonlinear switching characteristics of an NFET. $R_{P}$ and $R_{A P}$ result in different load lines. Since $\mathrm{R}_{\mathrm{AP}}$ has a higher resistance, the operating point during a read (point A) shows a lower current and lower voltage across the access device $\left(\mathrm{V}_{\mathrm{AC}}\right)$. A region of uncertainty can be overlaid onto the load lines. When the regions overlap due to $\mathrm{R}_{\mathrm{PAR}}$, incorrect sensing can occur. The overlap condition occurs when $\min \left(\mathrm{I}_{\mathrm{R}}(\mathrm{P})\right)<\max \left(\mathrm{I}_{\mathrm{R}}(\mathrm{AP})\right)$ which corresponds to $A_{P A R}<1$. Fig. 5(b) shows that $A_{P A R}$ becomes smaller as number of MTJs per cell or number of cells 


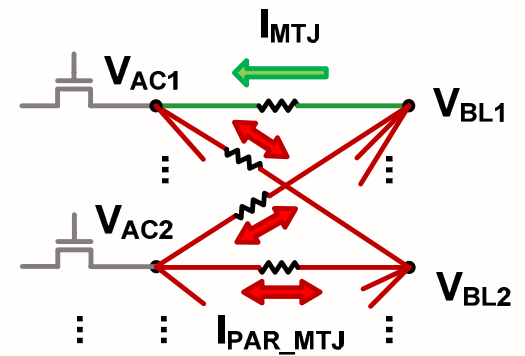

Fig. 6. Resistor network. Access transistors and bit-line drivers control every node voltage to properly control leakage current. However, this concept is practically unrealizable due to circuit complexity.
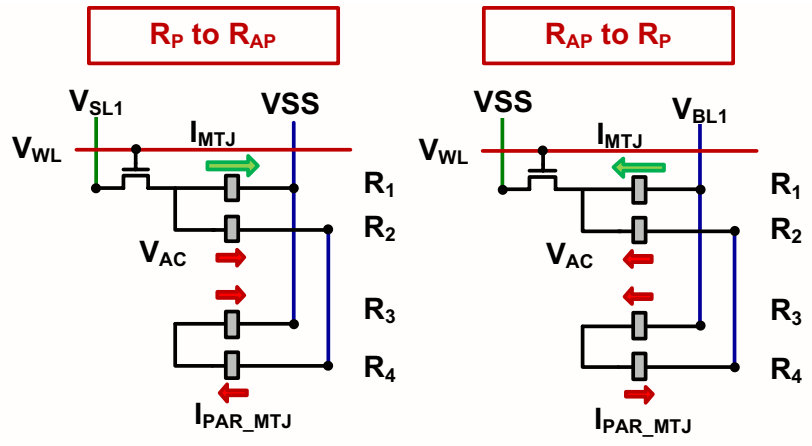

Fig. 7. Equivalent simplified model of memory array for (a) $R_{P}$ to $R_{A P}$ and (b) $R_{A P}$ to $R_{P}$ write access. Node-to-node impedance is max; therefore the leakage current is minimum. This optimistic case study sets the lower boundary of NFET size.

per column increases. Fig. 5(c) plots equation (2) to relate $A_{P A R}$

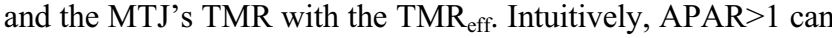
be maintained for small values of \#cells per column and \#MTJs per cell. Section III describes these limits in greater detail. For TMR greater than $100 \%$, a safe margin for successful read access can be achieved when the sub-array size (i.e. total number of MTJs per column) is kept below 9.

\section{B. Impact of MTJ Sharing on Writes}

Designing a cell with multiple MTJs for programming has two primary issues. First, writing a cell can result in an unintentional write of a non-accessed MTJ due to the parasitic current. Second, the parasitic paths can reduce the current through the accessed MTJ hence requiring a larger access transistor to provide the current.

A column of cells with multi-MTJ cells can be ideally modeled as a network of resistors (MTJs) as shown in Fig. 6. Each node can have an optimal voltage set such that the write current, $\mathrm{I}_{\mathrm{MTJ}}$, through the accessed MTJ is maximized and the $\mathrm{I}_{\text {PAR MTJ }}$ through any of the non-accessed MTJs is minimized. Since $I_{\text {PAR MTJ }}$ must be smaller than $\max I_{R}(A P)$ or $I_{R}(P)$ to avoid any probability of false switching, the ratio of the $\mathrm{I}_{\text {PAR MTJ }} / \mathrm{I}_{\text {MTJ }}$ determines the minimum ratio of $\max \left(\mathrm{I}_{\mathrm{R}}\right) / \min \left(\mathrm{I}_{\mathrm{W}}\right)$ required for the MTJ in order for a multi-MTJ cell to be possible. Actual implementation deviates from this lower bound depending on the ability to accurately set the individual node voltages. Section III addresses these cases. Analysis made here provides a lower bound.
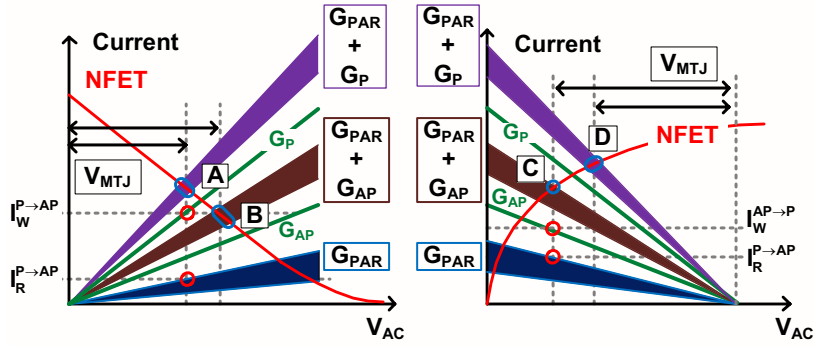

Fig. 8. (a) Switching current of ' 1 ' $\left(\mathrm{R}_{\mathrm{P}}\right.$ to $\left.\mathrm{R}_{\mathrm{AP}}\right)$ and Leakage current. At operating point A, the current through the selected MTJ should be greater than the minimum write current while the leakage current through any nonaccessed device should be smaller than the maximum read current. (b) Programming current of ' 0 ' $\left(\mathrm{R}_{\mathrm{AP}}\right.$ to $\left.\mathrm{R}_{\mathrm{P}}\right)$ and Leakage current.

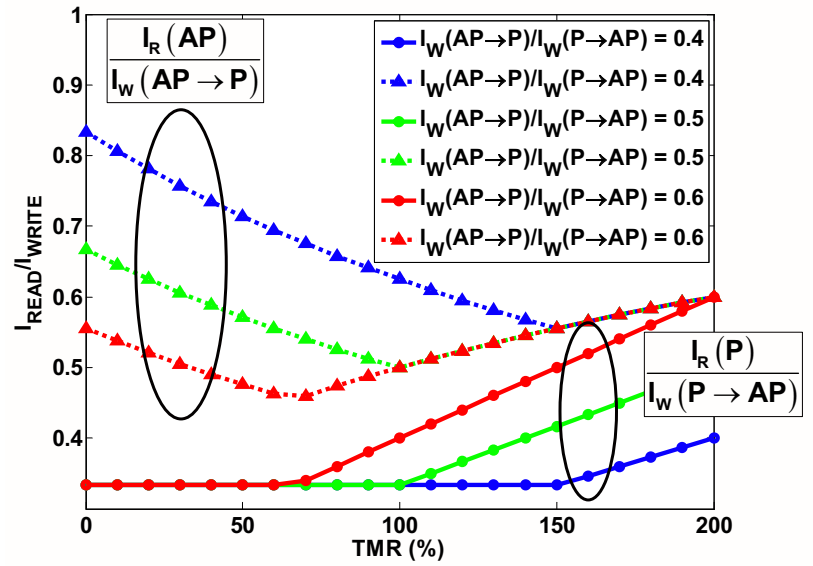

Fig. 9. Required ratio of minimum read current and maximum write current for $\mathrm{AP}$ to $\mathrm{P}$ and $\mathrm{P}$ to AP switching. As switching current is more asymmetric $(0.6 \rightarrow 0.4)$, the required current ratio of AP to $\mathrm{P}$ switching (dotted lines) is higher. The reverse relation can be seen from $\mathrm{P}$ to AP switching (solid lines).

Determining the minimum ratio for Fig. 6 is equivalent to a simplified model of 2 cells per column and 2 MTJs per cell as shown in Fig. 7. The worst-case conditions for writing $\mathrm{P} \rightarrow \mathrm{AP}$ and $\mathrm{AP} \rightarrow \mathrm{P}$ are shown. Writing a "1" $(\mathrm{P} \rightarrow \mathrm{AP})$ can be visualized graphically in Fig. $8(\mathrm{a})$ when the $\mathrm{I}_{\mathrm{MTJ}}$ at point $\mathrm{A}>$ $\min \left(\mathrm{I}_{\mathrm{W}}(\mathrm{P} \rightarrow \mathrm{AP})\right)$.

TABLE I.

THE WORST-CASE LEAKAGE DURING WRITE ACCESS

\begin{tabular}{|c|c|c|c|c|}
\hline \multicolumn{5}{|c|}{ Switching from $R_{P}$ to $R_{A P}$} \\
\hline$R 1$ & $R 2$ & $R 3$ & $R 4$ & Requirement \\
\hline $\mathrm{R}_{\mathrm{P}}$ & $\mathbf{R}_{\mathbf{P}}$ & $\mathbf{R}_{\mathbf{P}}$ & $\mathrm{R}_{\mathrm{P}}$ & $\frac{1}{3} \cdot I_{W R_{-} M I N}^{P \rightarrow A P}<I_{R E A D_{-} M A X}^{P}$ \\
\hline $\mathrm{R}_{\mathrm{P}}$ & $\mathrm{R}_{\mathrm{P}}$ & $\mathrm{R}_{\mathrm{P}}$ & $\mathbf{R}_{\mathrm{AP}}$ & $\frac{1}{(3+T M R)} \cdot I_{W R_{-} M I N}^{P \rightarrow_{A}}<I_{R E A D_{-} M A X}^{A P}$ \\
\hline \multicolumn{5}{|c|}{ Switching from $R_{A P}$ to $R_{P}$} \\
\hline$R 1$ & $R 2$ & $R 3$ & $R 4$ & Requirement \\
\hline $\mathrm{R}_{\mathrm{AP}}$ & $\mathrm{R}_{\mathrm{P}}$ & $\mathrm{R}_{\mathrm{P}}$ & $\mathbf{R}_{\mathbf{P}}$ & $\frac{(1+T M R)}{3} \cdot I_{W R_{-} M I N}^{A P \rightarrow P}<I_{R E A D_{-} M A X}^{P}$ \\
\hline $\mathrm{R}_{\mathrm{AP}}$ & $\mathrm{R}_{\mathrm{P}}$ & $\mathbf{R}_{\mathrm{AP}}$ & $\mathrm{R}_{\mathrm{P}}$ & $\frac{(1+T M R)}{(3+T M R)} \cdot I_{W R_{-} M I N}^{A P \rightarrow P}<I_{R E A D_{-} M A X}^{A P}$ \\
\hline
\end{tabular}


With this $\mathrm{I}_{\mathrm{MTJ}}$ through the accessed MTJ, the worst-case parasitic current can be defined as listed in Table I where R2 and R3 in the first case and R4 in the second case have a chance to be flipped if $I_{\text {PAR_MTJ }}>\max \left(I_{R}\right)$. When resistances through the parasitic path are equal, the ratio of $\max \left(\mathrm{I}_{\mathrm{R}}\right) / \min \left(\mathrm{I}_{\mathrm{W}}\right)$ is roughly $1 / 3$. As shown in the Table $\mathrm{I}$, the value can depend TMR since the programmed value of the non-accessed MTJ can vary between $R_{P}$ or $R_{A P}$. Fig. $8(b)$ and Table I show the worst case and ratio for writing a " 0 " $(\mathrm{AP} \rightarrow \mathrm{P})$. Fig. 9 visualizes requirements listed in Table I. As $\mathrm{I}_{\mathrm{W}}$ becomes highly asymmetric, the minimum ratio of read and write current should be higher. This plot indicates that the worst-case condition is generally dominated by maximum read current of $\mathrm{R}_{\mathrm{AP}}$.

The current through the parasitic resistance paths determine the additional amount of current that must be sourced by the access transistor. The lower bound of roughly $1 / 3$ indicates that the access device must be increased by at least $1 / 3$ of $\min \left(\mathrm{I}_{\mathrm{W}}(\mathrm{P} \rightarrow \mathrm{AP})\right)$ for each additional $\mathrm{MTJ} /$ cell.

Depending on the ability to control the voltages at each node, a higher ratio may be required which implies that the given MTJ has very narrow state transition region over control current [8]. The results in the next section assume the least complex implementation where nodes $\mathrm{V}_{\mathrm{AC} 2}$ in Fig. 6 are not driven to a specific voltage when the cell is not accessed.

\section{Simulation Results}

Feasibility and performace of using multi-MTJ cells that share a single access transistor depends on MTJ characteristics. Our analysis optimizes the area of the cell in a multi-MTJ design using an MTJ with characteristics as indicated in Table II. Values in the table are based on measured results of recent publications $[8,9]$ with adjustable read/write current ratio. The cell area is minimized based on reducing the required access device size for writing. The minimum device size is determined by adjusting bit-line voltages to meet the design targets given in Table II. Maximum parasitic current of non-accessed MTJ is carefully tested under various worst case conditions to remain within the MTJ specification. For this simulation, a $45 \mathrm{~nm}$ CMOS technology is used with HSPICE.

TABLE II. SIMULATED MTJ SPECS

\begin{tabular}{|c|c|}
\hline \multicolumn{2}{|c|}{ MTJ specifications } \\
\hline Terms & Value \\
\hline $\mathrm{R}_{\mathrm{A}}$ & $4.9 \Omega \cdot \mu \mathrm{m}^{2}$ \\
\hline $\mathrm{R}_{\mathrm{P}}$ & $750 \Omega$ \\
\hline $\mathrm{TMR}$ & $120(\%)$ \\
\hline $\begin{array}{c}\text { Switching Current } \\
\left(\mathrm{I}_{\mathrm{W}}(\mathrm{P} \rightarrow \mathrm{AP})\right)\end{array}$ & $500 \mu \mathrm{A}$ \\
\hline $\begin{array}{c}\text { Switching Current } \\
\left(\mathrm{I}_{\mathrm{W}}(\mathrm{AP} \rightarrow \mathrm{P})\right)\end{array}$ & $375 \mu \mathrm{A}$ \\
\hline $\begin{array}{c}\text { Max }\left(\mathrm{I}_{\mathrm{R}}(\mathrm{P})\right. \\
/ \min \left(\mathrm{I}_{\mathrm{W}}(\mathrm{P} \rightarrow \mathrm{AP})\right)\end{array}$ & 0.55 \\
\hline $\begin{array}{c}\text { Max }\left(\mathrm{I}_{\mathrm{R}}(\mathrm{AP})\right. \\
/ \min \left(\mathrm{I}_{\mathrm{W}}(\mathrm{AP} \rightarrow \mathrm{P})\right)\end{array}$ & $0.6 \sim 0.8$ \\
\hline
\end{tabular}

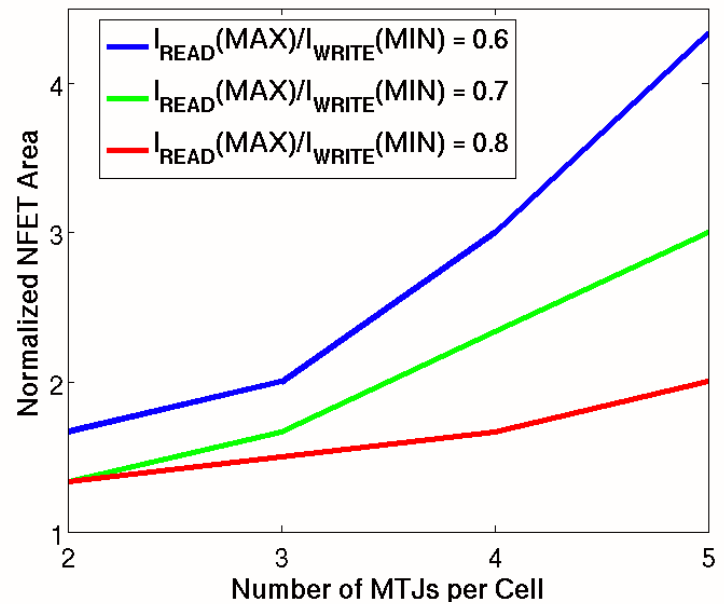

Fig. 10. NFET Area (normalized to 1T-1MTJ device size) as a function of number of MTJs per cell. Number of cells per column is fixed at 2. Higher tolerable read current lowers sensitivity of design space.

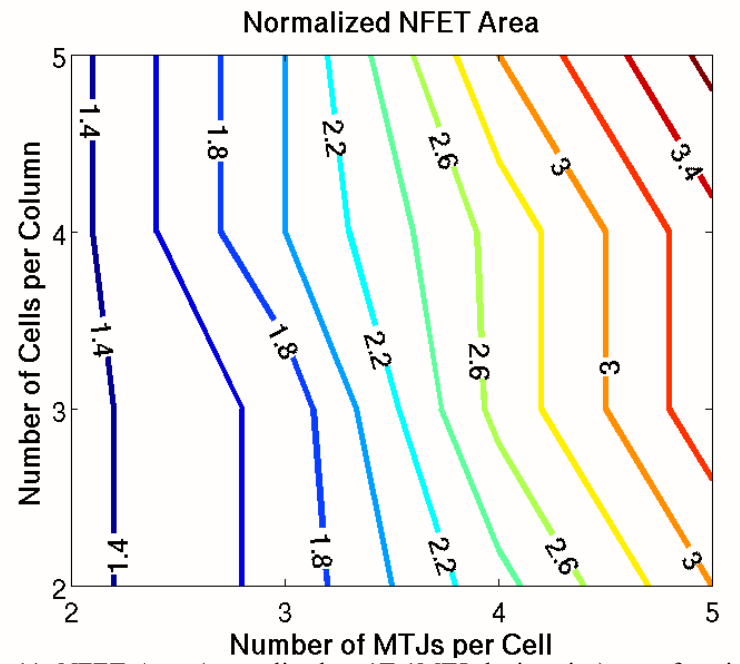

Fig. 11. NFET Area (normalized to 1T-1MTJ device size) as a function of number of MTJs per cell and number of cells in the sub-array. The number of cells per column has relatively small impact on the NFET size as long as bitline drivers have controls on the leakage current. The $\max (\mathrm{read}) / \min ($ write) current ratio used in this plot is 0.7 .

Our analysis explores the impact of the shared design on cell area and effective TMR for various numbers of MTJs per cell and numbers of cells per column. The simulation results of the shared structure are normalized by the 1T-1MTJ cell area.

\section{A. Normalized NFET Size}

Figure 10 shows normalized device area of the access transistor as a function of \# MTJ per cell with different ratios of $\max \left(\mathrm{I}_{\mathrm{R}}(\mathrm{AP})\right) / \min \left(\mathrm{I}_{\mathrm{W}}(\mathrm{AP} \rightarrow \mathrm{P})\right)$. With a large number of MTJs per cell, the parasitic path has low impedance leading to higher parasitic currents. Hence, the bit-lines associated with the non-accessed cells should be driven as strongly as possible to avoid sizing up the access NFET unnecessarily. An inherent tradeoff is present with the $\max \left(\mathrm{I}_{\mathrm{R}}(\mathrm{AP})\right) / \min \left(\mathrm{I}_{\mathrm{W}}(\mathrm{AP} \rightarrow \mathrm{P})\right.$ ratio 


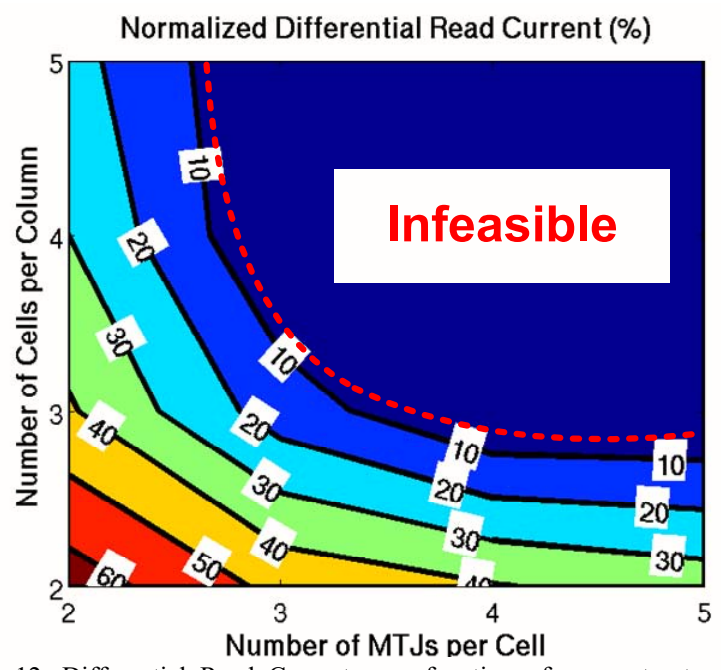

Fig. 12. Differential Read Current as a function of array structure. The $\max (\mathrm{read}) / \mathrm{min}$ (write) current ratio used in this plot is 0.7 .

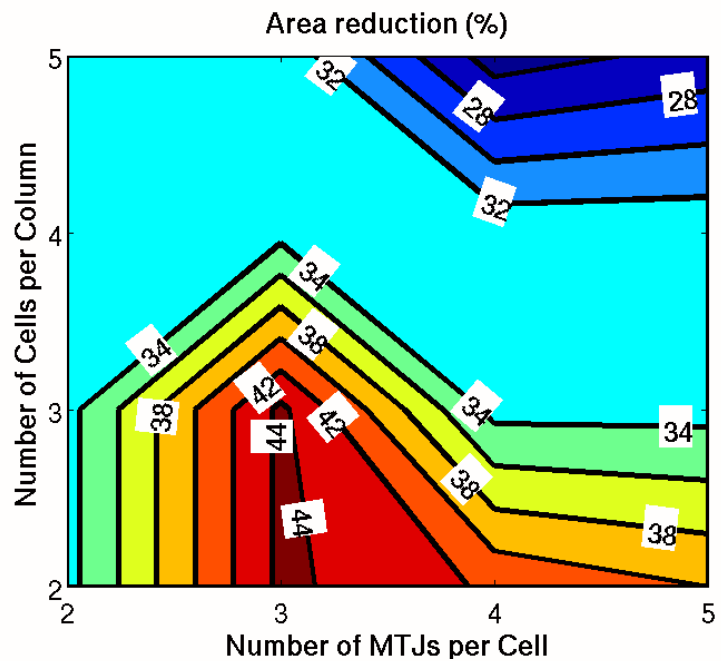

Fig. 13. Cell area reduction per MTJ compared to 1T-1MTJ device size. The $\max (\mathrm{read}) / \mathrm{min}$ (write) current ratio used in this plot is 0.7 .

and the amount of parasitic current. As shown in the figure, if the node at the access device of a non-accessed cell is not driven, the ratio must increase to be greater than 0.5 for the multi-MTJ structure to be feasible. The slope of each curve increases with larger numbers of MTJs/cell because the nonaccessed nodes (nodes $\mathrm{V}_{\mathrm{AC} 2}$ in Fig. 6) are not simultaneously driven. The larger number of parasitic paths in parallel with increasing number of $\mathrm{MTJ} /$ cell results in greater parasitic current through the non-accessed devices. Hence, the area efficiency degrades.

Fig. 11 sweeps the NFET size as a function of the number of cells per column (i.e. sub-array size). The sub-array size has little impact on the NFET size as long as net parasitic resistance is greater than the source impedance of bit-line driver. Once bit-line driver loses control over idle bit-lines, however, the performance worsens considerably where the parasitic current cannot be properly controlled. Equivalently, if the non-accessed bit-lines are floating, the amount of NFET current is roughly proportional to the number of MTJs per cell and is very sensitive to the number of cells per column.

\section{B. Simulated Differential Read Current}

As indicated by the effective TMR in Equation (2), parasitic resistors desensitize resistance variation of target MTJ. Fig. 12 shows that the differential read current is always less than $60 \%$ of $1 \mathrm{~T}-1 \mathrm{MTJ}$ case. In this simulation, the access device size has been scaled as in Fig. 11. The results indicate that the differential current drops substantially with increasing number of MTJs per cell and hence realistically only 2 or 3 MTJs per cell is functionally feasible. With larger numbers of cells per column, we anticipate the differential read current to be $<20 \%$ of the $1 \mathrm{~T}-1 \mathrm{MTJ}$ case putting more demand on the sensing circuitry or longer time for sensing.

\section{Area Saving}

Assuming that access transistor size is a dominant factor of memory density, cell area reduction rate per MTJ is shown in Fig. 13. Note that this ratio is normalized to cell area of 1T$1 \mathrm{MTJ}$ case. This figure clearly indicates that higher memory density can be attained though the cost comes from reduced sensing margin and increased risk of data loss. Table III summarizes simulation results.

TABLE III. SIMULATION RESULTS

\begin{tabular}{|c|c|}
\hline Terms & value \\
\hline Number of MTJs per Cell & 3 \\
\hline Number of Cells & 3 \\
\hline Switching Current (P $\rightarrow \mathrm{AP})$ & $500 \mu \mathrm{A}$ \\
\hline Switching Current (AP $\rightarrow \mathrm{P})$ & $375 \mu \mathrm{A}$ \\
\hline $\begin{array}{c}\text { Max Read Current/ Min } \\
\text { Switching Current }\end{array}$ & $70 \%$ \\
\hline $\begin{array}{c}\text { Differential Read Current } \\
\text { (Normalized) }\end{array}$ & $15 \%$ \\
\hline $\begin{array}{c}\text { Cell area per MTJ } \\
\text { (Normalized) }\end{array}$ & $56 \%$ \\
\hline
\end{tabular}

\section{CONCLUSION}

This paper describes the impact of using multiple MTJs that shares a single access device in a cell. Because of the resistive nature of the MTJs, device sharing has substantial limitations due to the parasitic resistive paths that degrade both the read differential current and write stability. The technique promises modest improvements in the array density but can only be applied to small number of MTJs per cell and limited column size. Furthermore, MTJ characteristics must have a high $\max \left(\mathrm{I}_{\mathrm{R}}\right) / \min \left(\mathrm{I}_{\mathrm{W}}\right)$ ratio in order to avoid instability of nonaccessed MTJs. 


\section{ACKNOWLEDGMENT}

This work was supported by the DARPA/MTO STT-RAM program. The authors would like to thank UCLA STT-RAM team for helpful discussions and for providing measurement data.

\section{REFERENCES}

[1] C. Chappert, et al., "The Emergence of Spin Electronics in Data Storage", Nature Materials, June 2007, pp. 813-823.

[2] M. Hosomi, et al., "A Novel Nonvolatile Memory with Spin Torque Transfer Magnetization Switching: Spin-RAM", IEEE IEDM, Dec. 2005, pp. 459-462.

[3] A. Raychowdhury, et al., "Design Space and Scalability Exploration of 1T-1STT MTJ Memory Arrays in the Presence of Variability and
Disturbances," in Electron Devices Meeting (IEDM), 2009 IEEE International, December 2009, pp. 1-4.

[4] J. DeBrosse, et al., "1 $16 \mathrm{Mb}$ RAM featuring bootstrapped write drivers", Symp. VLSI Technol., p. 454, 2004

[5] F. Z. Wang, "Diode-free magnetic random access memobyr using spindependent tunneling effect," Appl. Phys. Lett., vol. 77, no. 13, pp. 20362038, 2000 .

[6] N. Sakimura, et al., "A 512Kb Cross-Point Cell MRAM," in IEEE ISSCC Dig. Tech. Papers, 2003, pp. 278-279.

[7] C.J. Amsinck, et al., "Scaling constraints in nanoelectronic randomaccess memories," Nanotechnol., vol. 16, no. 10, pp. 2251-2260, 2005.

[8] P.K. Amiri, et al., "Low Write-Energy Magnetic Tunnel Junctions for High-Speed Spin-Transfer-Torque RAM", IEEE Electron Device Letters, January 2011, Vol. 32, Issue 1, pp. 57-59

[9] Z. M. Zeng, et al., "Effect of resistance-area product on spin-transfer switching in MgO-based magnetic tunnel junction memory cells", Appl. Phys. Lett. 98, 072512, 2011 